\title{
Alteration of cingulate long-term plasticity and behavioral sensitization to inflammation by environmental enrichment
}

\author{
Fanny W.F. Shum, ${ }^{1}$ Long-Jun Wu, ${ }^{1}$ Ming-Gao Zhao, ${ }^{1}$ Hiroki Toyoda, ${ }^{1}$ Hui Xu, ${ }^{1}$ \\ Ming Ren, ${ }^{1}$ Raphael Pinaud, ${ }^{1}$ Shanelle W. Ko, ${ }^{1}$ Yong-Seok Lee, ${ }^{2}$ Bong-Kiun Kaang, ${ }^{2}$ \\ and Min Zhuo ${ }^{1,3}$ \\ ${ }^{1}$ Department of Physiology, Faculty of Medicine, University of Toronto, University of Toronto Centre for the Study of Pain, \\ 1 King's College Circle, University of Toronto, Toronto M5S 1A8, Canada; ${ }^{2}$ Department of Biological Sciences, \\ College of Natural Sciences, Seoul National University, Seoul 151-742, South Korea
}

\begin{abstract}
Exposure to an enriched environment (EE) has been shown to induce cortical plasticity. Considerable amount of research is focused on the effects of EE in the hippocampus; however, effects of EE on other brain regions and the mechanisms involved are not well known. To investigate this, we induced cortical plasticity by placing mice in an EE for one month and measured the effects of EE in the anterior cingulate cortex (ACC). Here, we show that EE enhanced the expression of the plasticity gene, egr-l, in the ACC of EE animals accompanied by enhanced cingulate long-term potentiation (LTP) and decreased cingulate long-term depression (LTD). The increased NMDA receptor NR2B/NR2A subunits current ratio is associated with the plasticity seen in the ACC while total protein levels remain unchanged. Furthermore, behavioral experiments show that these mice exposed to EE demonstrate enhanced responses to acute and long-term inflammation. Our findings suggest that exposure to EE alters physiological properties within the ACC which results in enhanced responses to inflammation.
\end{abstract}

Environmental enrichment is known to increase cortical plasticity and is one of the most reliable and well characterized paradigms of experience-dependent plasticity in rodents. This experimental paradigm has been repeatedly shown to trigger widespread morphological changes in the mammalian brain (Walsh et al. 1969; Rosenzweig and Bennett 1972; Diamond et al. 1976) such as neurogenesis (Kempermann et al. 1997), increased dendritic arborizations (Globus et al. 1973; Greenough et al. 1973; Green et al. 1983), synaptic contacts and neurotransmission (Greenough et al. 1978; Altschuler 1979), and elevated immediate early gene expression (Wallace et al. 1995). A vast amount of studies have focused on the positive effects of environmental enrichment in brain regions involved in learning and memory such as the hippocampus and neocortex (van Praag et al. 2000). For example, enriched animals exhibit enhanced hippocampal long-term potentiation (LTP) (Duffy et al. 2001), enhanced performance in hippocamppal-dependent behavioral task in the Morris water maze (Kempermann et al. 1997; Williams et al. 2001), and in fear conditioning (Duffy et al. 2001). Furthermore, exposure to enriched environments (EE) has been shown to enhance plasticity and functional recovery associated with cerebral insult (Dahlqvist et al. 1999; Rampon et al. 2000), suggesting environmental enrichment triggers cortical reorganization leading to functional recovery after injury. Despite the well reported effects of environmental enrichment in the hippocampus and neocortex, less is known about the changes induced by EE in other brain regions.

The anterior cingulate cortex (ACC) participates in cognitive functions including higher order emotional responses and several forms of memory (Bush et al. 2000; Han et al. 2003;

\footnotetext{
${ }^{3}$ Corresponding author.
}

E-mail min.zhuo@utoronto.ca; fax: (416) 978-4940.

Article is online at http://www.learnmem.org/cgi/doi/10.1101//m.530607.
Frankland et al. 2004; Tang et al. 2005). In addition to the roles of the ACC in memory, processing at this level also plays a key role in regulating central nervous system responses to peripheral and central injuries (Davis et al. 1997; Zhuo 2004). For example, peripheral inflammation caused enhanced presynaptic glutamate release (Zhao et al. 2006) as well as the up-regulation of postsynaptic NR2B (Wu et al. 2005b). Digit amputation results in bilateral plastic neural changes in the ACC, including an increase in immediate early gene expression, such as $c$-fos, egr-1, and CREB (Wei et al. 1999), and increased electrophysiological responses (Wei et al. 2001; Wu et al. 2005a). The ACC is involved in both the sensory and emotional component of behavioral sensitization to injury. For example, electric or chemical activation of the ACC produced fear memory (Tang et al. 2005) and aversive learning (Johansen and Fields 2004). However, the extent to which EE affects the information processed within the ACC remains unknown.

Exposure of animals to an EE triggers a robust Egr-1 expression, especially over sensory cortices, where the highest density of morphological changes occurs as a function of experience (Wallace et al. 1995; Pinaud 2004). The activity-dependent immediate early gene (IEG) egr-1 (also called NGFI-A, Krox24, or zif/268) has been repeatedly used as a mapping tool for neuronal activity, with single-cell resolution (Milbrandt 1987; Cole et al. 1989; Wallace et al. 1995) and has also been proposed to be a key component of a cascade of events involved in the process of cortical plasticity. Furthermore egr-1 expression has been shown to depend on $N$-methyl- $D$-aspartate (NMDA) receptor activation (Cole et al. 1989) and to be required for the maintenance of late phase LTP (Jones et al. 2001; Ko et al. 2005a).

The NMDA receptor plays a crucial role in synaptic plasticity and the induction of LTP (Bliss and Collingridge 1993) in many brain regions including the hippocampus and ACC. Enriched animals display enhanced hippocampal LTP (Duffy et al. 2001) 
and increased NR2A and NR2B NMDA receptor subunit expression in the forebrain (Tang et al. 2001). Functionally, mice with overexpressed NR2B subunits display increased response to tissue injury and inflammation (Wei et al. 2001). The cellular mechanism of NMDA receptor-mediated plasticity is dependent on the $\mathrm{NR} 2 \mathrm{~B} / \mathrm{NR} 2 \mathrm{~A}$ subunit composition ratio in the ACC, and not in the hippocampus (Zhao et al. 2005).

Here, we examined the effects of EE on synaptic plasticity changes in the ACC and the extent to which enhanced sensory experience affects behavioral sensitization to injury. First, we used an activity marker Egr-1 to determine the regulation of gene expression after enrichment. To investigate the involvement of NMDA receptors in the ACC after enrichment, we induced LTP and long-term depression (LTD) and also determined the NR2B/ NR2A current composition ratio in enriched mice. Furthermore, altered synaptic plasticity in the cortex results in better learning and memory in parallel with increased behavioral response to inflammation (Ji et al. 2003), suggesting that pain and memory share similar molecular mechanisms. Therefore, since exposure to EE enhanced memory performances in animals, we hypothesize that these animals may also exhibit enhanced behavioral responses to inflammation.

\section{Results}

\section{Expression of egr-1 in the ACC}

Since egr-1 expression reflects neuronal activity, we investigated whether egr-1 expression is altered as a function of sensory experience. Immunohistochemistry was used to compare egr-1 protein expression in the ACC of animals exposed to EE versus age-matched controls. Lamination of the ACC was determined by Nissl staining (Fig. 1D). In controls, a low density of immunolabeled cell nuclei was detected throughout all ACC areas and cortical layers, although the highest density of egr-1 positive cells was observed in the superficial layer (II) (Fig. 1C,D). This basal Egr-1 expression pattern observed in our control animals is in accordance with previous reports on the distribution of egr-1 mRNA and protein in the prefrontal cortex (Wallace et al. 1995; Pinaud et al. 2002). Conversely, a robust egr-1 induction was detected in the ACC of animals exposed to EE (Fig. 1B). This marked increase in the number of egr-1 immunolabeled nuclei was evident for all cortical layers and was more pronounced in the superficial and middle layers (III), as compared to deep layers (V and VI) (Fig. 1D,E). Corresponding to the immunohistochemistry data, Egr-1 protein expression in the ACC of enriched animals was significantly increased compared to controls (control: $100.4 \pm 21.7$; enriched: $167.7 \pm 16.1, n=4$ per group, $P<0.01$ ) (Fig. 1F). These results indicate that exposure of animals to a plasticity-inducing paradigm triggers the expression of an activity-dependent gene in the ACC and further suggests that connectivity within this region may be altered as a function of sensory experience.

\section{Normal basal synaptic transmission, but enhanced LTP and decreased LTD in the ACC of enriched mice}

We next asked whether EE also triggered synaptic modifications in the ACC circuitry. To address this question, we performed whole-cell patch clamp recordings on ACC slice preparations of enriched animals after 4 wk of EE exposure and control animals. Recordings were obtained from pyramidal neurons located in layers II/III of the ACC, which were identified both by visual inspection and by the action potential profile that resulted from the injection of depolarizing current. Typically, pyramidal neurons in the supragranular layers of the ACC show significant firing frequency adaptation (Zhao et al. 2005). First, we wanted to know whether the exposure of animals to an EE affected basal synaptic transmission in the ACC. To address this question, we studied non-NMDA receptor-mediated basic excitatory postsynaptic currents (EPSCs) in control and enriched mice. In the ACC, most of non-NMDA receptor-mediated basal synaptic responses are mediated by AMPA receptors (Wu et al. 2005c). The input-output relationship of EPSCs in enriched mice $(n=10)$ was similar to those in control mice $(n=10$, $P>0.05)$ at various stimulus intensities (Fig. 2A). To examine the voltage dependence of EPSCs, the currents were recorded at different holding potentials from $-70 \mathrm{mV}$ to $+50 \mathrm{mV}$ with $20 \mathrm{mV}$ steps. The current-voltage (I-V) relationships of EPSCs were then plotted. No difference was found in the I-V relationship of basal EPSCs between enriched mice $(n=9)$ and control mice $(n=7$, $P>0.05$ ) (Fig. 2B).

Next, we tested whether exposure of animals to an EE impacted synaptic plasticity in our preparations by investigating the formation of LTP and LTD. Pairing presynaptic activation with postsynaptic spikes that occurred at an interval of $10 \mathrm{msec}$ resulted in a significant long-lasting potentiation of synaptic responses in both control (Bi and Poo 1998; Zhao et al. 2005) and enriched mice (Fig. 3B). Interestingly, a comparison between control and EE groups revealed a significant increase in LTP that occurred in the EE group (control: $120.5 \pm 9.4 \%, n=5$; enriched: $145.4 \pm 9.4 \%, n=7 ; P<0.01$ ) (Fig. 3B,C). Next, LTD was examined in control and enriched mice. The protocol induced significant LTD in the ACC of

Figure 1. Environmental enrichment enhanced Egr-1 expression in the ACC. (A) Diagrammatic representation of ACC and surrounding areas in the mouse brain. Egr-1 immunolabeled cells in ACC of enriched animals $(B)$ were significantly increased compared to control animals $(C)$. (D) Cresyl violet stained section showing the lamination of the ACC. (E) Summary data of the number of Egr-1 positive cells in a $100 \times 100 \mu \mathrm{m}$ sample in superficial, middle, and deep layers of ACC. *, Significant differences were detected between superficial (II) and middle (III), but not deep, layers ( $\mathrm{V}$ and $\mathrm{VI}) ; P<0.05$. $(F$ Western blot of Egr-1 protein expression in the ACC of enriched animals was significantly increased compared to controls. Scale bars: $(A) 1 \mathrm{~mm} ;(B-D) 200 \mu \mathrm{m}$. Error bars indicate SEM. 
A

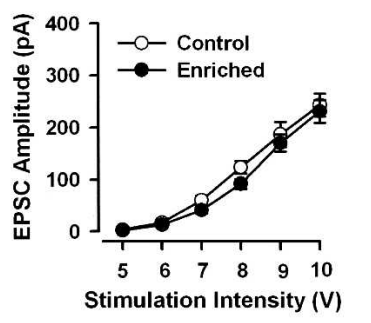

B

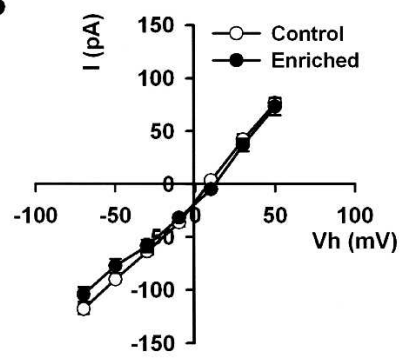

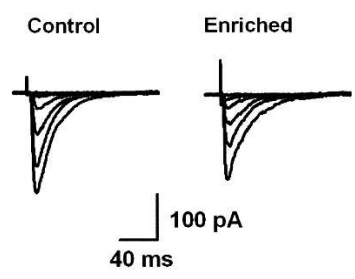

Control

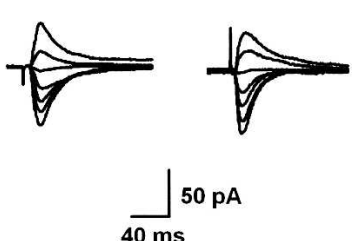

Figure 2. Normal non-NMDA receptor-mediated basal synaptic transmission in enriched mice. In the ACC, most of non-NMDA receptormediated basal synaptic responses are mediated by AMPA receptors. $(A)$ Input-output relationship for AMPA receptor-mediated EPSCs in control $(n=10)$ and enriched mice $(n=10)$. Input-output relationship for AMPA receptor-mediated EPSCs in the enriched mice is unaltered as compared with wild-type mice. $(B)$ No difference of current-voltage plots for AMPA receptor-mediated EPSCs between control $(n=7)$ and enriched mice $(n=9)$.

control mice (78.6 $\pm 4.3 \%, n=10 ; P<0.05)$ (Fig. 3D,E), similar to our previous report using 300 pulses at $1 \mathrm{~Hz}$ paired with postsynaptic depolarization at $-45 \mathrm{mV}$ (Toyoda et al. 2005). However, LTD was largely decreased or diminished in enriched mice (95.4 $\pm 6.5 \%, n=7 ; P<0.05)$ (Fig. 3D,E).

Presynaptic glutamate release is unaltered in the ACC Both presynaptic and postsynaptic mechanisms have been proposed to contribute to the expression of synaptic plasticity (Nicoll and Malenka 1995). To determine whether the altered plasticity observed in EE animals is a result of presynaptic modifications, we conducted paired stimulation protocols. Two stimuli were delivered with an interval ranging from 35 to $150 \mathrm{msec}$ in duration. The short interval between the stimuli resulted in a facilitation of the second response, which was referred to as the paired-pulse facilitation (PPF). Our results showed that PPF was similar between control and EE mice for all intervals observed (control: $n=9$; enriched: $n=9$; $P>0.05$ ) (Fig. 4B). Next, to investigate whether an increase in the readily releasable pool of vesicles would account for the altered plasticity observed in EE mice, we depolarized ACC synapses using a protocol of repetitive stimulation at physiological frequencies at $10 \mathrm{~Hz}$ for $30 \mathrm{sec}$ (Rosahl et al. 1995). This stimulation protocol, which causes a continuous decline in neurotransmitter release, resulted in a rapid and pronounced activity-dependent depression. However, no differences were detected when comparing the results obtained in ACC slices from EE and control animals (Fig. 4C,D). These results suggest that altered plasticity observed in animals exposed to the EE setting was unlikely to result from presynaptic mechanisms.

\section{Enhanced NR2B:NR2A ratio in NMDA receptor-mediated EPSCs}

Based on our previous findings, we hypothesized that EE-induced alteration of LTP and LTD observed in our preparations could be mediated through a dynamic regulation of specific NMDA receptor subunits in the ACC. To test this hypothesis, we first pharmacologically isolated NMDAR-mediated EPSCs in ACC neurons in control and EE-exposed mice. With local stimulation in the ACC, a slow-decaying inward EPSC was recorded in the presence of picrotoxin $(50 \mu \mathrm{M})$ and CNQX $(10 \mu \mathrm{M})$. The decay time constant $(\tau)$ of EPSCs in EE-exposed mice was significantly prolonged in comparison with control mice (control: $90.0 \pm 3.0 \mathrm{msec}$, $n=7$; enriched: $104.0 \pm 3.4 \mathrm{msec}, n=13 ; P<0.05)$. This indicates that the component of NR2B-containing NMDA receptors is enhanced in EE-exposed mice. Next, we selectively blocked NR2A or NR2B subunits by bath-applying antagonists NVP-AAM077 and Ro25-6981, respectively, and examined synaptically induced NMDAR-mediated EPSCs. We then calculated the relative ratios

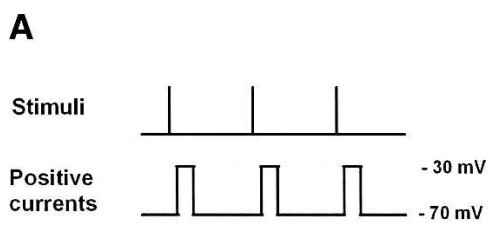

\section{B}

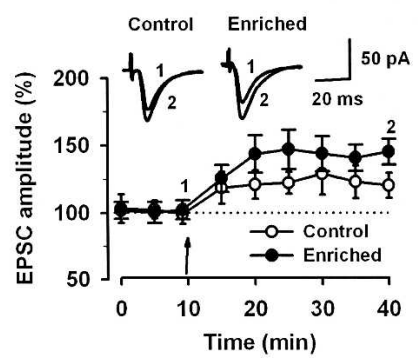

C

D
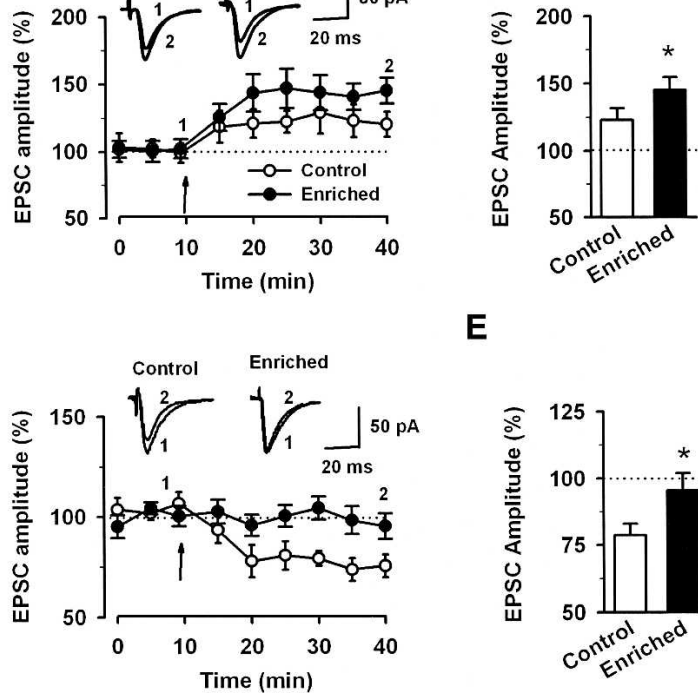

$\mathbf{E}$

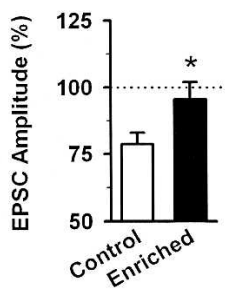

Figure 3. Enrichment alters synaptic properties of ACC neurons. (A) Schematic illustrating the induction protocol involving the pairing of three presynaptic stimuli which caused three EPSPs with three postsynaptic action potentials at $30 \mathrm{~Hz}$, paired 15 times every $5 \mathrm{sec}$. (B) LTP was induced by paired training with EPSP $10 \mathrm{msec}$ ahead of postsynaptic action potentials in the ACC. The enriched animals showed enhanced EPSC amplitude before and $30 \mathrm{~min}$ after training. EPSC responses were averaged over 5-min intervals. Traces show averages of EPSCs 5 min before (1) and 30 min after (2) the paired training (arrow). (C) Summary result of EPSC amplitude 30 min after LTP induction was significantly increased in mice exposed to EE compared with control mice. (D) LTD was induced by paired training with EPSP $10 \mathrm{msec}$ after postsynaptic action potentials in the ACC. Traces show averages of EPSCs 5 min before (1) and 30 min after (2) the paired training (arrow). (E) Summary result of EPSC amplitude 30 min after LTD induction was significantly decreased in mice exposed to EE compared with control mice. ${ }^{\star} P<0.05$, compared with control group. Error bars indicate SEM. 
A

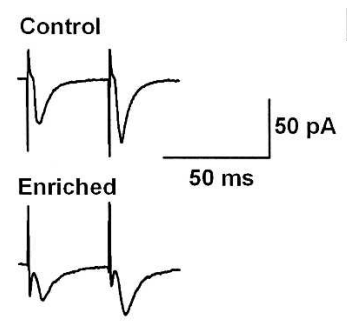

C

B

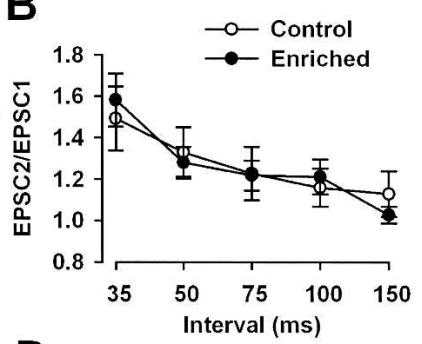

D Enriched
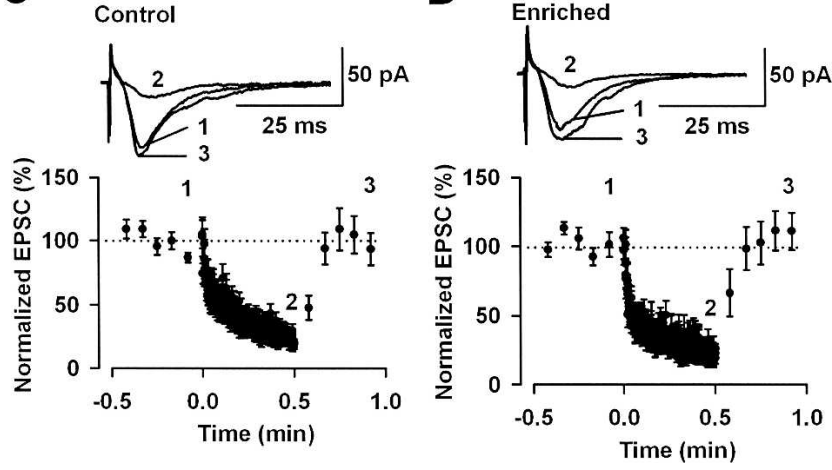

Figure 4. Presynaptic effects in the ACC after the enrichment. (A) Sample PPF traces at an interval of $50 \mathrm{msec}$. (B) PPF in all five intervals was unchanged between control and enriched animals $(n=9$ for each group). (C,D) Similar responses to a $10-\mathrm{Hz}$ repetitive stimulation in ACC synapses between control and enriched animals. (Insert) Traces show the basal EPSC amplitude (1), amplitude at the end of repetitive stimulation (2), recovered EPSC amplitude after repetitive stimulation (3). $P>0.05$, compared with control group. Error bars indicate SEM.

of NR2A- and NR2B-mediated currents after pharmacological intervention and compared those results against total baseline NMDAR-mediated EPSCs. NVP-AAM077 treatment significantly reduced the total NMDAR-mediated currents in undisturbed control, as compared to EE-exposed mice (control: $82.6 \pm 1.0 \%$, $n=7$; enriched: $72.9 \pm 1.7 \%, n=13 ; P<0.05)$ (Fig. 5A). Interestingly, sequential perfusion of slices with Ro25-6981 induced a further reduction in NMDAR-mediated currents in EE-exposed mice as compared to control mice (control: $6.5 \pm 0.8 \%, n=7$; enriched: $10.9 \pm 0.8 \%, n=13 ; P<0.05$ ) (Fig. $5 \mathrm{~A}$ ), suggesting that enhanced NR2B expression is regulated by experience in the ACC. This effect becomes evident when a ratio NR2B/NR2A is generated by comparing the Ro25-6981 sensitive EPSCs to NVPAAM077 sensitive EPSCs. The NR2B/NR2A ratio was higher in EE-exposed, as compared to control, mice $(0.15 \pm 0.01$ vs. $0.08 \pm 0.01$, respectively; $P<0.05$ ) (Fig. 5B). These results suggest that sensory experience changed synaptic plasticity in the ACC by altering the dynamic regulation of NMDA receptor subunits. To test whether the expression of NR2B and NR2A protein was altered after the EE, Western blotting was used to estimate the amount of different NMDA type 2 subunits (Fig. 5C). We did not find any significant change in the total amount of protein (control, $n=3$; EE, $n=3 ; P>0.05$ ) or phosphorylated protein (control, $n=3$; EE, $n=3 ; P>0.05$ ) of NR2B or NR2A in enriched mice compared with that of control mice (Fig. 5C).

\section{Behavioral sensitization to injury after enrichment}

To determine if EE affects behavioral responses to noxious stimulus, we first measured responses to nociceptive spinal reflex. We found no significant changes in the tail-flick response latency (control: $5.3 \pm 0.3 \mathrm{sec}, n=10$; enriched: $4.9 \pm 0.3 \mathrm{sec}, n=13$; $P>0.05)$, which indicates that spinal transmission was unaltered

after EE. Similarly, response latency to a noxious heat stimulus measured by placing the mouse on a hotplate set to $55^{\circ} \mathrm{C}$ was not significantly altered between control $(11.5 \pm 1.0 \mathrm{sec}, n=10$; $P>0.05)$ and enriched (9.9 $\pm 0.8 \mathrm{sec}, n=13 ; P>0.05)$ mice.

To determine if enrichment alters behavioral responses to short-term and long-term injury, we injected formalin subcutaneously into the dorsal side of a hind paw. This injection has been shown to cause three phases of licking responses over 120 min after the injection (Wei et al. 2001). The first phase of the formalin test has been shown to reflect stimulation of primary afferent fibers and the second phase is a measurement of peripheral inflammatory responses (Tjolsen et al. 1992), while the third phase responses have been shown to measure the enhanced longterm activity-dependent synaptic plasticity (Tjolsen et al. 1992; Kim et al. 1999; Wei et al. 2001). Within the first 55 min after formalin injection, behavioral responses were similar between control and enriched mice (Fig. 6A). However, during the third phase (55-120 $\mathrm{min}$ ) of the formalin licking response, the enriched group $(n=8)$ exhibited significantly more licking as compared to control mice ( $n=7, P<0.01)$ (Fig. $6 \mathrm{~B})$. Since the third phase of the formalin test represents the long-term effects of NMDA receptor-mediated synaptic activity at central synapses, it is reasonable to see an enhanced third phase response in enriched animals. These results suggest that EE experience alters signal pathways in the ACC, which is involved in the sensitivity to acute injury.

To further explore EE-induced changes in the behavioral responses to chronic injury, we carried out mechanical allodynia after hind paw injection of complete Freund's adjuvant (CFA). Application of a von Frey fiber to the dorsum of a hind paw before CFA injection elicited no responses. At 1, 3, and $7 \mathrm{~d}$ after

A

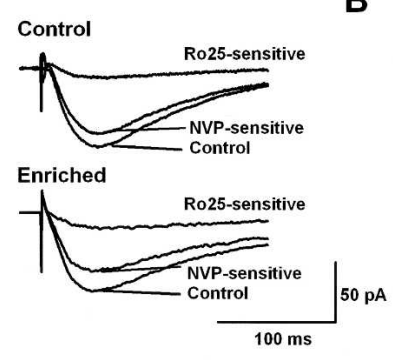

C

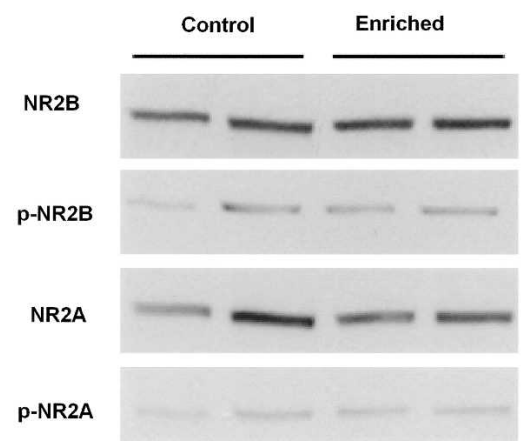

Figure 5. Contribution of NMDA receptor subunits in ACC after the enrichment. (A) Representative traces show NR2A or NR2B componentmediated EPSC. NVP-AAM077 sensitive trace represents NR2A component and Ro25-6981 sensitive as the NR2B component. (B) Summary of the NR2B/NR2A current ratio in control and enriched animals. The NR2B/ NR2A current ratio is enhanced in enriched animals. ${ }^{* *} P<0.01$, compared with control mice. Error bars indicate SEM. (C) Western blotting showing that there is no difference in total protein or phosphorylation of NR2B between control and enriched group. Similarly, no difference was found for NR2A subunit. 

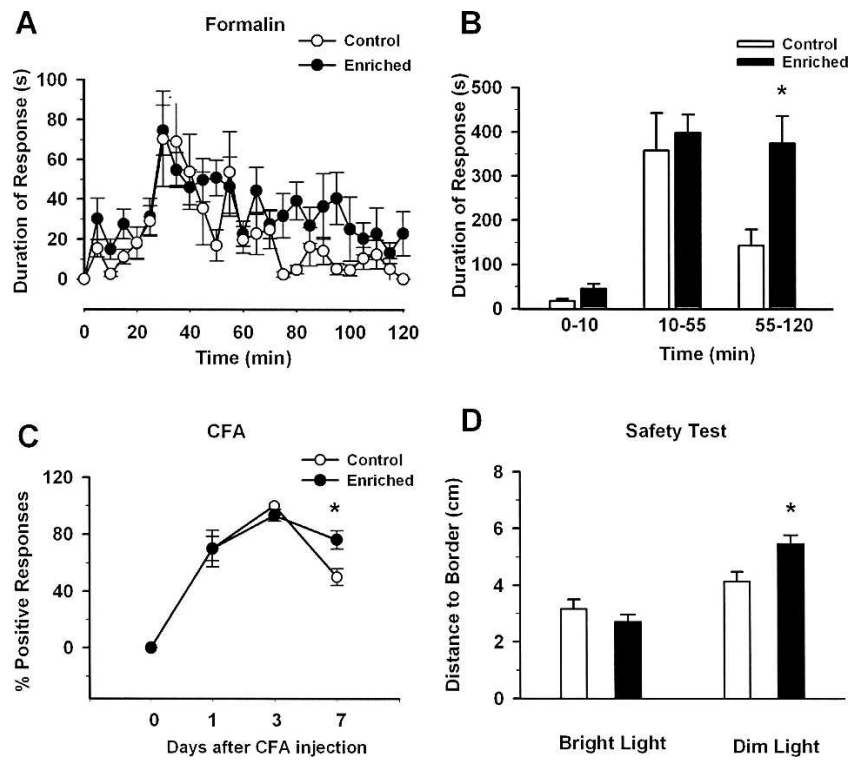

Figure 6. Enrichment enhanced behavioral sensitivity to safety and injury responses. (A) Enriched and control animals were injected with $10 \mu \mathrm{L}$ of formalin (5\%), in the right hind paw and were monitored over a time course of $2 \mathrm{~h}$ corresponding to the three phases of formalin-induced response. Enriched animals showed significantly enhanced licking response during the third phase $(B)$ compared with control animals. (C) Timeline showing $10 \mu \mathrm{L}$ CFA (50\%) injection into hind paw of enriched animals enhanced chronic injury response on $7 \mathrm{~d}$ in ipsilateral paw. (D) Enriched animals spent more time away from the border of the arena indicating an increased response to the safety signal. ${ }^{*} P<0.05$, compared with control group. Error bar indicates SEM.

CFA injection $(50 \%, 10 \mu \mathrm{L})$, mice responded to the same fiber by hind paw withdrawal. We found that mechanical allodynia on day 1 and 3 was similar in mice that were exposed to EE and age-matched undisturbed mice. Interestingly, on day 7 , mice exposed to EE showed enhanced sensitivity to the von Frey fiber compared with control animals (Fig. 6C), indicating that enriched animals developed greater sensitivity to chronic inflammation. Taken together, these results clearly suggest that exposure to an enriched environment can enhance behavioral sensitivity to acute and prolonged inflammatory pain.

Innate safety processing is altered in enriched animals Emotional learning, such as fear, involves higher brain centers such as the ACC, amygdala, hippocampus, and other cortical areas. Recent studies have suggested that changes in synaptic transmissions are important for the establishment and consolidation of different forms of fear memory (Han et al. 2003; Zhao et al. 2005). While much has been explored in the area of fear, less is known about the brain regions responsible for the sense of safety. Due to the lack of paradigms used to investigate the sense of safety, much less is known about the responses to a safe environment. One test developed to measure an animal's sense of safety is an unlearned safety paradigm using bright and dim illuminations (Rogan et al. 2005). Bright light elicits an unlearned or instinctive fear response, and reduced illumination elicits an instinctive safety signal in mice. Enriched and control mice explored the open field area for $1 \mathrm{~min}$ at 500 lux before the luminance was reduced to 1 lux. Mice typically walk along the borders of a novel open field to reduce the chances of encounter predators and make occasional runs to the center of the arena. We found that under bright illumination, both enriched and control animals kept to the walls of the arena (Fig. 6D). After the intro- duction of a dimmed ambient illumination (1 lux) both groups of animals responded to the dimmed light as a safety signal and increased the exploration time in the center of the arena (Fig. 6D). We observed that, under dimmed lighting, enriched animals spent more time exploring away from the border of the arena when compared to the control animals (Fig. 6D). Furthermore, to rule out the possibility of hyper-locomotor activities in enriched animals, the total distance traveled within $30 \mathrm{~min}$ in the open field was measured. Our results show that there was no difference in the distance traveled between the two groups (control, $45.7 \pm 6.2 \mathrm{~m}, n=6$; enriched, $52.5 \pm 1.7 \mathrm{~m}, n=8 ; P>0.05)$. Our results suggest that enriched animals were equally stressed in novel environments and that the increased distance to the border under dim light is the result due to increased exploratory activities in enriched mice.

\section{Discussion}

In this study, we examined the consequences of enhanced sensory experience in the ACC by placing animals in an enriched environment. We demonstrate that exposure of animals to an EE results in increased egr- 1 expression in the ACC accompanied by enhanced LTP and decreased LTD. The shift in NR2B/NR2A subunit-mediated currents suggests that enhanced sensory experience is reflected in vitro via alterations in NMDA receptor current composition. These synaptic changes may underlie the enhanced sensitization to acute and chronic inflammation without affecting the sensitivity to acute noxious stimuli. We are the first to observe these changes in the ACC after exposing to environmental enrichment, and our present findings provide several new findings regarding to the role of the ACC after EE.

Studies from human imaging showed that the ACC is activated by different factors such as motivational drive (Shima and Tanji 1998), attention (Posner 1994), and response to inflammation (Rainville et al. 1997). Despite the vast amount of studies concentrating on the plasticity of the ACC, these studies have only explored the consequences of the plasticity change in the ACC after inflammation (Wei et al. 1999; Wu et al. 2005b). Because the brain is dynamic, it is not surprising to find that modifications in the ACC occur during pathological conditions such as injury. In our study, we used an environmental enrichment paradigm to evoke responses in the ACC. One of the major advantages of using $\mathrm{EE}$ as a stimulus to investigate plasticity changes in the ACC is that it does not involve injuring the animal.

Neuronal functions are thought to be dependent on gene expression. The external stimuli to cells, such as depolarization or activation of NMDA receptors, are known to trigger the expression of IEGs (Ghosh et al. 1994), which in turn activates a cascade of downstream gene expression. One of such IEG is Egr-1, an activity-dependent zinc finger transcription factor (Milbrandt 1987; Lemaire et al. 1988; O'Donovan et al. 1999) responsible for coupling extracellular changes to cellular functions, where it plays a role in the expression of genes that possess the Egr-1 binding domain in their promoters. The association between Egr-1 activation, NMDA receptors, and LTP has been known for many years (Cole et al. 1989). Studies have shown an upregulation of Egr-1 mRNA after LTP induction in several brain regions including the insular cortex (Jones et al. 1999), dentate gyrus, amygdale, and hippocampus. More recently, evidence suggests that Egr-1 mRNA is up-regulated in the hippocampus after exposure to a novel stimulus (Hall et al. 2000), and the deletion of Egr-1 in mice resulted in decreased long-term synaptic and behavioral plasticity in the hippocampus (Jones et al. 2001; Ko et al. 2005a).

Using Egr-1 as a marker for neuronal activity, we saw en- 
hanced and sustained levels of egr-1 gene expression in animals exposed to EE. Typically, protein levels of IEGs such as Egr-1 are found to be at basal levels but undergo a dramatic increase upon $\mathrm{EE}$ and returns to basal levels within a few hours after each episode of EE (Wallace et al. 1995). The sustained increase in Egr-1 expression coincides with enhanced ACC LTP and may further contribute to the increase in behavioral sensitization to injury. We have previously reported that egr- 1 expression in the ACC is enhanced after injury (Ko et al. 2005b). Conversely, our results show that Egr-1 expression triggered by EE also results in enhanced sensitivity to injury. From these results, we speculate that the sensitivity to inflammation occurs after cortical plasticity changes at the level of ACC and such changes lead to an increase in the activation of NMDA receptor function, which is enhanced in parallel to Egr-1 activation.

The ACC is part of the thalamo-limbic-cortex circuitry where it receives various sensory inputs from the thalamus and sends outputs to several brain regions such as the hippocampus and amygdala. Here, we further investigated the ACC-related behavioral consequences of environmental enrichment. Because the ACC plays an important role in responses to injury (Wei et al. 2001), we asked whether the change in the ACC after EE would alter the sensitivity to injury. Our results found that enriched mice showed enhanced responses to peripheral inflammation, in the formalin and CFA model. Therefore, these results support our hypothesis that EE-induced plasticity change in the ACC leads to the altered behavioral sensitization to injury. There are several lines of evidence showing that the ACC has positive role in mediating nociceptive responses: (1) Electric or chemical activation of the ACC significantly facilitates the spinal nociceptive tailflick reflex (Calejesan et al. 2000). Excitation of the ACC produced fear memory (Tang et al. 2005) and aversive learning (Johansen and Fields 2004). (2) Inhibition of excitatory synaptic transmission by opiates in the ACC (Tanaka and North 1994; Wei et al. 1999) or lesion of the ACC reduced nociceptive hotplate responses and inhibited behavioral nociceptive responses to formalin inflammation (Lee et al. 1999). (3) Forebrain-targeted NR2B overexpression including the ACC increased behavioral sensitization responses to injury (Wei et al. 2001). Moreover, peripheral inflammation up-regulated NR2B in the ACC and local inhibition of NR2B in the ACC reduced inflammation-induced nociception (Wu et al. 2005b). In sum, this evidence points out that the ACC is involved in the nociceptive responses, and NMDA NR2B plays a critical role in the ACC-related physiological and pathological function such as persistent pain. The underlying mechanism may be due to the involvement of NR2B in synaptic plasticity in the ACC (Toyoda et al. 2005; Zhao et al. 2005). Therefore, our results suggest that the enhanced sensitivity to injury may be mediated by an increase in NMDAR subunit NR2B current ratios in the ACC after EE. However, we cannot rule out the involvement of the many downstream target genes affected by enhanced NMDA receptor function in the ACC that may contribute to the behaviors we see in enriched animals.

We have to mention that our results showed the enhancement in NR2B:NR2A current ratio in the adult ACC was not accompanied by increased NR2B subunits expression in the enriched animal. These results suggested the function of NR2Bcontaining NMDA receptor was enhanced without the increase in total number of NR2B receptors. Since the phosphorylation of NR2B is not altered in the enriched mice, there are two possibilities for functional change in NR2B subunit: One explanation is that more NR2B-containing NMDA receptor is trafficked to the membrane after EE; alternatively, the EE may result in increased amounts of functional NR2B-containing NMDA receptors move from extrasynaptic or perisynaptic sites to synaptic sites. Future experiments are needed to further reveal signaling pathways for contributing to enhanced NMDA receptor-mediated responses.

As noted above, environmental enrichment has a variety of effects on cellular functions and behavioral responses. In terms of synaptic plasticity, most results from different laboratories agreed that enrichment results in enhanced synaptic plasticity (Foster et al. 1996; Duffy et al. 2001; Artola et al. 2006). Although our results clearly showed that the EE altered synaptic plasticity in the ACC as well as ACC-related function, such as behavioral sensitization to peripheral inflammation, different results of the $\mathrm{EE}$ on synaptic plasticity and behaviors were reported in other brain areas by different laboratories (van Praag et al. 2000; Nithianantharajah and Hannan 2006). For example, some reports showed the enhanced EPSP (Green and Greenough 1986; Foster et al. 1996) whereas others did not observe any change (van Praag et al. 1999). Different results were also found in the role of neurogenesis in behavior outputs in enriched mice (Bruel-Jungerman et al. 2005; Meshi et al. 2006). The reason for the variability may be mainly due to the different environmental enrichment methods and different brain regions, as well as experimental preparations (Nithianantharajah and Hannan 2006). It is also known that strain and species differences are the major factors in the outcome of behavioral responses following EE (van de Weerd et al. 1994). In this study, we enriched mice of C57/BL6 background, a strain which has been shown to have positive effects after EE such as decreased anxiety-like behavior.

Using environmental enrichment, we demonstrated experience-dependent plasticity in the ACC. We found that environmental enrichment leads to an up-regulation of NR2B subunitmediated currents, accompanied by altered long-term synaptic plasticity and increased behavioral sensitivity to injury. Our results shed new light onto how the brain responds to external stimuli at the level of the ACC, and increases our understanding of the functional consequences of EE. Understanding how experience affects plasticity in the brain will increase our understanding of the mechanisms altered in response to memory and injury.

\section{Materials and Methods}

\section{Animals}

All subjects were $4 \mathrm{wk}$ of age at the start of the experiment. C57BL/6 mice were purchased from Charles River. Upon arrival, animals were weighed and ear-marked and assigned to either standard or enriched housing. Mice were housed on a 12-h lightdark schedule with food and water available ad libitum. All experiments were carried out in accordance with the rules and regulations of the Animal Care and Use Committee at the University of Toronto.

\section{Environmental enrichment}

Mice were divided into two groups: control and enriched. Controls were group housed under standard conditions but were not exposed to enrichment chamber. The environmental enrichment consists of a spacious cage (Habitrail) with various toys, tunnels, platforms, spin wheels, ladders. The arrangement of these objects was changed everyday to make the environment novel for mice. During the enrichment period, mice were allowed to explore the enrichment environment for $2 \mathrm{~h}$ per day continuously for $4 \mathrm{wk}$. All experiments were conducted after 4 wk of enrichment period.

\section{Immunohistochemistry}

Brain sections were prepared on a cryostat and incubated with egr-1 (1:500; Santa Cruz Biotechnology). Briefly, the sections were incubated at room temperature in a blocking buffer solution $(0.5 \%$ albumin and $0.3 \%$ Triton $\mathrm{X}-100$ in $0.1 \mathrm{M}$ PBS) and incubated overnight at $4^{\circ} \mathrm{C}$ with anti-Egr- 1 . Sections were then incu- 
bated for $2 \mathrm{~h}$ at room temperature with a biotinylated goat antirabbit IgG (1:200; Chemicon). For DAB reaction, sections were processed with the Vector $\mathrm{ABC}$ kit (Vector Laboratories). For Nissl staining, sections were placed in a $0.5 \%$ Cresyl violet solution for 5 min, dehydrated in a series of alcohols, defatted in xylenes, and coverslipped.

\section{Slice preparation}

Coronal brain slices $(300 \mu \mathrm{M})$ from $6-8$-wk-old mice, containing the ACC, were prepared using standard methods (Wu et al. 2005c). Slices were transferred to a submerged recovery chamber containing oxygenated $\left(95 \% \mathrm{O}_{2}\right.$ and $\left.5 \% \mathrm{CO}_{2}\right)$ artificial cerebrospinal fluid (ACSF) (in mM: $124 \mathrm{NaCl}, 2.5 \mathrm{KCl}, 2 \mathrm{CaCl}_{2}, 1 \mathrm{MgSO}_{4}$, $25 \mathrm{NaHCO}_{3}, 1 \mathrm{NaH}_{2} \mathrm{PO}_{4}, 10$ glucose) at room temperature for at least $1 \mathrm{~h}$.

\section{Electrophysiology}

Experiments were performed in a recording chamber on the stage of an Axioskop 2FS microscope with infrared DIC optics for visualizing whole-cell patch clamp recordings. Excitatory postsynaptic currents (EPSCs) were recorded from layer II-III neurons using an Axon 200B amplifier (Axon Instruments) and stimulations were delivered using a bipolar tungsten stimulating electrode placed in layer V of the ACC. AMPAR-mediated EPSCs were induced by repetitive stimulations at $0.02 \mathrm{~Hz}$ and neurons were voltage-clamped at $-70 \mathrm{mV}$. The recording pipettes (3-5 megaohms) were filled with solution containing (mM) $145 \mathrm{~K}$ gluconate, $5 \mathrm{NaCl}, 1 \mathrm{MgCl}_{2}, 0.2$ EGTA, 10 HEPES, $2 \mathrm{Mg}$-ATP, and $0.1 \mathrm{Na}_{3}$-GTP (adjusted to pH 7.2 with $\mathrm{KOH}$ ). LTP was induced within 12 min after obtaining stable EPSCs to prevent the washout effect. To induce LTP, the protocol includes two steps: (1) A train of stimuli: each presynaptic stimulation was applied 10 msec ahead of postsynaptic action potentials (APs) for three times, $30 \mathrm{~Hz}$; (2) the train stimulation was then applied 15 times every $5 \mathrm{sec}$. The induction protocol for LTD was similar to that for LTP, except that the presynaptic stimulation was applied 10 msec after postsynaptic APs. Paired stimulation protocols with intervals ranging from 35 to $150 \mathrm{msec}$ in duration were used to study the presynaptic release probability. The NMDAR-mediated component of EPSCs was pharmacologically isolated in ACSF containing CNQX $(20 \mu \mathrm{M})$ and picrotoxin $(100 \mu \mathrm{M})$. The patch electrodes contained (in mM) 102 cesium gluconate, 5 TEAchloride, $3.7 \mathrm{NaCl}$, 0.2 EGTA, 20 HEPES, 2 Mg-ATP, 0.3 Na-GTP, and 5 QX-314 chloride (adjusted to $\mathrm{pH} 7.2$ with $\mathrm{CsOH}$ ). Neurons were voltage-clamped at $-30 \mathrm{mV}$, and NMDARs-mediated EPSCs were evoked at $0.05 \mathrm{~Hz}$. NVP-AAM077 and Ro25-6981 were bathapplied sequentially to assess the NR2A and NR2B components of EPCSs. The access resistance was 15-30 megaohms and was monitored throughout the experiment. Data were discarded if access resistance changed by $>15 \%$ during an experiment. Results are expressed as means \pm SEM. Statistical comparisons were performed using the Student $t$-test. Experiments were conducted "blind" to the group assignments.

\section{Behavioral experiments}

To test acute pain responses, the latency of response to heating of the tail (tail-flick test) or to placement on a hotplate $\left(55^{\circ} \mathrm{C}\right)$ was measured as described (Calejesan et al. 2000). To test inflammatory pain, formalin $(5 \%, 10 \mu \mathrm{L}$; Sigma) or complete Freund's adjuvant (50\% in saline, $10 \mu \mathrm{L}$; Sigma) was injected subcutaneously into the dorsal side of a hind paw. For formalin-induced responses, the total time spent licking or biting the injected hind paw was recorded during each five-minute interval, for two hours. To test for CFA-induced responses, mechanical sensitivity was assessed with a set of von Frey filaments. We used a filament that was found to be innocuous before the CFA injection to detect mechanical allodynia. The filament was applied to the point of bending six times each to the dorsal surfaces of the left and right hind paw. Positive responses included prolonged hind paw withdrawal followed by licking or scratching. For each time point, the percentage response frequency of hind paw withdrawal was expressed as follows: (number of positive responses)/
$6 \times 100$ per hind paw. To test the safety response in mice, each mouse was placed in a circular arena adapted from Rogan et al. (2005) and was allowed to explore the arena in bright illumination (500 lux) for $1 \mathrm{~min}$, and the illumination was decreased to ambient lighting ( 1 lux) for $1 \mathrm{~min}$. The total distance traveled and the distance to the wall of the area were measured. In all cases, experiments were conducted "blind" to observers.

\section{Western blot and immunoprecipitation}

Equal amounts of protein from the ACC were separated and electrotransferred onto PDVF membranes (Invitrogen), which were probed with anti-Egr-1 (Santa Cruz), anti-NR2A (Chemicon), anti-NR2B (Chemicon), with $\beta$-actin (Sigma) as a loading control. The membranes were incubated with horseradish peroxidaseconjugated secondary antibody (anti-rabbit IgG), and bands were visualized using an ECL system (Perkin Elmer). For immunoprecipitation, the solubilized proteins were diluted 20 -fold with modified RIPA buffer $(50 \mathrm{mM}$ Tris- $\mathrm{HCl}$ at $\mathrm{pH} 7.4,1 \% \mathrm{NP}-40$, $0.25 \%$ Na-deoxycholate, $150 \mathrm{mM} \mathrm{NaCl}, 1 \mathrm{mM}$ EDTA, $1 \mathrm{mM}$ PMSF) and incubated with $50 \mu \mathrm{L}$ of protein G-agarose precoupled with anti-phosphotyrosine antibody (PY20, BD Biosciences) for $3 \mathrm{~h}$ at $4^{\circ} \mathrm{C}$. The reaction mixtures were then washed three times and eluted by boiling in sample loading buffer and subjected to Western blot as described above. Results are expressed as means \pm SEM and were analyzed using Scion Image software. Statistical comparisons were performed using the $t$-test or the paired $t$-test.

\section{Data analysis and statistics}

Results were analyzed by $t$-test, paired $t$-test, and TWO WAY ANOVA followed by post-hoc Student-Newman-Keuls test to identify significant differences. All data are expressed as mean \pm SEM. In all cases, $P<0.05$ was considered statistically significant.

\section{Acknowledgments}

We thank Wilson Yu, Eva Knifed, and Susan Kim for their assistance with enrichment and behavior testing. This work was supported by grants from the EJLB-Canadian Institutes of Health Research Michael Smith Chair in Neurosciences and Mental Health, the Canada Research Chair, and the Canadian Institute for Health Research and National Institutes of Health-National Institute of Neurological Disorders and Stroke Grant NS42722 (M.Z.). L.-J.W. is supported by post-doc fellowships from Canadian Institutes of Health Research and the Fragile X Foundation of Canada. B.-K.K. was supported in part by National Research Laboratory, Brain Research Center-Frontier Program, Korea.

\section{References}

Altschuler, R.A. 1979. Morphometry of the effect of increased experience and training on synaptic density in area CA3 of the rat hippocampus. J. Histochem. Cytochem. 27: 1548-1550.

Artola, A., von Frijtag, J.C., Fermont, P.C., Gispen, W.H., Schrama, L.H., Kamal, A., and Spruijt, B.M. 2006. Long-lasting modulation of the induction of LTD and LTP in rat hippocampal CA1 by behavioural stress and environmental enrichment. Eur. J. Neurosci. 23: 261-272.

Bi, G.Q. and Poo, M.M. 1998. Synaptic modifications in cultured hippocampal neurons: Dependence on spike timing, synaptic strength, and postsynaptic cell type. J. Neurosci. 18: 10464-10472.

Bliss, T.V. and Collingridge, G.L. 1993. A synaptic model of memory: Long-term potentiation in the hippocampus. Nature 361: 31-39.

Bruel-Jungerman, E., Laroche, S., and Rampon, C. 2005. New neurons in the dentate gyrus are involved in the expression of enhanced long-term memory following environmental enrichment. Eur. J. Neurosci. 21: 513-521.

Bush, G., Luu, P., and Posner, M.I. 2000. Cognitive and emotional influences in anterior cingulate cortex. Trends Cogn. Sci. 4: 215-222.

Calejesan, A.A., Kim, S.J., and Zhuo, M. 2000. Descending facilitatory modulation of a behavioral nociceptive response by stimulation in the adult rat anterior cingulate cortex. Eur. J. Pain 4: 83-96.

Cole, A.J., Saffen, D.W., Baraban, J.M., and Worley, P.F. 1989. Rapid increase of an immediate early gene messenger RNA in hippocampal neurons by synaptic NMDA receptor activation. Nature 340: 474476. 
Dahlqvist, P., Zhao, L., Johansson, I.M., Mattsson, B., Johansson, B.B., Seckl, J.R., and Olsson, T. 1999. Environmental enrichment alters nerve growth factor-induced gene A and glucocorticoid receptor messenger RNA expression after middle cerebral artery occlusion in rats. Neuroscience 93: 527-535.

Davis, K.D., Taylor, S.J., Crawley, A.P., Wood, M.L., and Mikulis, D.J. 1997. Functional MRI of pain- and attention-related activations in the human cingulate cortex. J. Neurophysiol. 77: 3370-3380.

Diamond, M.C., Ingham, C.A., Johnson, R.E., Bennett, E.L., and Rosenzweig, M.R. 1976. Effects of environment on morphology of rat cerebral cortex and hippocampus. J. Neurobiol. 7: 75-85.

Duffy, S.N., Craddock, K.J., Abel, T., and Nguyen, P.V. 2001. Environmental enrichment modifies the PKA-dependence of hippocampal LTP and improves hippocampus-dependent memory. Learn. Mem. 8: 26-34.

Foster, T.C., Gagne, J., and Massicotte, G. 1996. Mechanism of altered synaptic strength due to experience: Relation to long-term potentiation. Brain Res. 736: 243-250.

Frankland, P.W., Bontempi, B., Talton, L.E., Kaczmarek, L., and Silva, A.J. 2004. The involvement of the anterior cingulate cortex in remote contextual fear memory. Science 304: 881883.

Ghosh, A., Ginty, D.D., Bading, H., and Greenberg, M.E. 1994. Calcium regulation of gene expression in neuronal cells. J. Neurobiol. 25: 294-303.

Globus, A., Rosenzweig, M.R., Bennett, E.L., and Diamond, M.C. 1973. Effects of differential experience on dendritic spine counts in rat cerebral cortex. J. Comp. Physiol. Psychol. 82: 175-181.

Green, E.J. and Greenough, W.T. 1986. Altered synaptic transmission in dentate gyrus of rats reared in complex environments: Evidence from hippocampal slices maintained in vitro. J. Neurophysiol. 55: 739-750

Green, E.J., Greenough, W.T., and Schlumpf, B.E. 1983. Effects of complex or isolated environments on cortical dendrites of middle-aged rats. Brain Res. 264: 233-240.

Greenough, W.T., Volkmar, F.R., and Juraska, J.M. 1973. Effects of rearing complexity on dendritic branching in frontolateral and temporal cortex of the rat. Exp. Neurol. 41: 371-378.

Greenough, W.T., West, R.W., and DeVoogd, T.J. 1978. Subsynaptic plate perforations: Changes with age and experience in the rat. Science 202: 1096-1098.

Hall, J., Thomas, K.L., and Everitt, B.J. 2000. Rapid and selective induction of BDNF expression in the hippocampus during contextual learning. Nat. Neurosci. 3: 533-535.

Han, C.J., O'Tuathaigh, C.M., van Trigt, L., Quinn, J.J., Fanselow, M.S., Mongeau, R., Koch, C., and Anderson, D.J. 2003. Trace but not delay fear conditioning requires attention and the anterior cingulate cortex. Proc. Natl. Acad. Sci. 100: 13087-13092.

Ji, R.R., Kohno, T., Moore, K.A., and Woolf, C.J. 2003. Central sensitization and LTP: Do pain and memory share similar mechanisms? Trends Neurosci. 26: 696-705.

Johansen, J.P. and Fields, H.L. 2004. Glutamatergic activation of anterior cingulate cortex produces an aversive teaching signal. Nat. Neurosci. 7: 398-403.

Jones, M.W., French, P.J., Bliss, T.V., and Rosenblum, K. 1999. Molecular mechanisms of long-term potentiation in the insular cortex in vivo. J. Neurosci. 19: RC36.

Jones, M.W., Errington, M.L., French, P.J., Fine, A., Bliss, T.V. Garel, S., Charnay, P., Bozon, B., Laroche, S., and Davis, S. 2001. A requirement for the immediate early gene Zif268 in the expression of late LTP and long-term memories. Nat. Neurosci. 4: 289-296.

Kempermann, G., Kuhn, H.G., and Gage, F.H. 1997. More hippocampal neurons in adult mice living in an enriched environment. Nature 386: $493-495$.

Kim, S.J., Calejesan, A.A., Li, P., Wei, F., and Zhuo, M. 1999. Sex differences in late behavioral response to subcutaneous formalin injection in mice. Brain Res. 829: 185-189.

Ko, S.W., Ao, H.S., Mendel, A.G., Qiu, C.S., Wei, F., Milbrandt, J., and Zhuo, M. 2005a. Transcription factor Egr-1 is required for long-term fear memory and anxiety. Sheng Li Xue Bao 57: 421-432.

Ko, S.W., Vadakkan, K.I., Ao, H., Gallitano-Mendel, A., Wei, F. Milbrandt, J., and Zhuo, M. 2005b. Selective contribution of Egr1 (zif/268) to persistent inflammatory pain. J. Pain 6: 12-20.

Lee, D.E., Kim, S.J., and Zhuo, M. 1999. Comparison of behavioral responses to noxious cold and heat in mice. Brain Res. 845: 117121.

Lemaire, P., Revelant, O., Bravo, R., and Charnay, P. 1988. Two mouse genes encoding potential transcription factors with identical DNA-binding domains are activated by growth factors in cultured cells. Proc. Natl. Acad. Sci. 85: 4691-4695.

Meshi, D., Drew, M.R., Saxe, M., Ansorge, M.S., David, D., Santarelli, L., Malapani, C., Moore, H., and Hen, R. 2006. Hippocampal neurogenesis is not required for behavioral effects of environmental enrichment. Nat. Neurosci. 9: 729-731.

Milbrandt, J. 1987. A nerve growth factor-induced gene encodes a possible transcriptional regulatory factor. Science 238: 797-799.

Nicoll, R.A. and Malenka, R.C. 1995. Contrasting properties of two forms of long-term potentiation in the hippocampus. Nature 377: $115-118$.

Nithianantharajah, J. and Hannan, A.J. 2006. Enriched environments, experience-dependent plasticity and disorders of the nervous system. Nat. Rev. Neurosci. 7: 697-709.

O’Donovan, K.J., Tourtellotte, W.G., Millbrandt, J., and Baraban, J.M. 1999. The EGR family of transcription-regulatory factors: Progress at the interface of molecular and systems neuroscience. Trends Neurosci. 22: $167-173$.

Pinaud, R. 2004. Experience-dependent immediate early gene expression in the adult central nervous system: Evidence from enriched-environment studies. Int. J. Neurosci. 114: 321-333.

Pinaud, R., Tremere, L.A., Penner, M.R., Hess, F.F., Robertson, H.A. and Currie, R.W. 2002. Complexity of sensory environment drives the expression of candidate-plasticity gene, nerve growth factor induced-A. Neuroscience 112: 573-582.

Posner, M.I. 1994. Attention: The mechanisms of consciousness. Proc. Natl. Acad. Sci. 91: 7398-7403.

Rainville, P., Duncan, G.H., Price, D.D., Carrier, B., and Bushnell, M.C 1997. Pain affect encoded in human anterior cingulate but not somatosensory cortex. Science 277: 968-971.

Rampon, C., Tang, Y.P., Goodhouse, J., Shimizu, E., Kyin, M., and Tsien, J.Z. 2000. Enrichment induces structural changes and recovery from nonspatial memory deficits in CA1 NMDAR1-knockout mice. Nat. Neurosci. 3: 238-244.

Rogan, M.T., Leon, K.S., Perez, D.L., and Kandel, E.R. 2005. Distinct neural signatures for safety and danger in the amygdala and striatum of the mouse. Neuron 46: 309-320.

Rosahl, T.W., Spillane, D., Missler, M., Herz, J., Selig, D.K., Wolff, J.R., Hammer, R.E., Malenka, R.C., and Sudhof, T.C. 1995. Essential functions of synapsins I and II in synaptic vesicle regulation. Nature 375: 488-493.

Rosenzweig, M.R. and Bennett, E.L. 1972. Cerebral changes in rats exposed individually to an enriched environment. J. Comp. Physiol. Psychol. 80: 304-313.

Shima, K. and Tanji, J. 1998. Role for cingulate motor area cells in voluntary movement selection based on reward. Science 282: 13351338.

Tanaka, E. and North, R.A. 1994. Opioid actions on rat anterior cingulate cortex neurons in vitro. J. Neurosci. 14: 1106-1113.

Tang, Y.P., Wang, H., Feng, R., Kyin, M., and Tsien, J.Z. 2001. Differential effects of enrichment on learning and memory function in NR2B transgenic mice. Neuropharmacology 41: 779-790.

Tang, J., Ko, S., Ding, H.K., Qiu, C.S., Calejesan, A.A., and Zhuo, M. 2005. Pavlovian fear memory induced by activation in the anterior cingulate cortex. Mol. Pain 1: 6 .

Tjolsen, A., Berge, O.G., Hunskaar, S., Rosland, J.H., and Hole, K. 1992. The formalin test: An evaluation of the method. Pain 51: 5-17.

Toyoda, H., Zhao, M.G., and Zhuo, M. 2005. Roles of NMDA receptor NR2A and NR2B subtypes for long-term depression in the anterior cingulate cortex. Eur. J. Neurosci. 22: 485-494.

van de Weerd, H.A., Baumans, V., Koolhaas, J.M., and van Zutphen, L.F. 1994. Strain specific behavioural response to environmental enrichment in the mouse. J. Exp. Anim. Sci. 36: 117-127.

van Praag, H., Christie, B.R., Sejnowski, T.J., and Gage, F.H. 1999 Running enhances neurogenesis, learning, and long-term potentiation in mice. Proc. Natl. Acad. Sci. 96: 13427-13431.

van Praag, H., Kempermann, G., and Gage, F.H. 2000. Neural consequences of environmental enrichment. Nat. Rev. Neurosci. 1: $191-198$.

Wallace, C.S., Withers, G.S., Weiler, I.J., George, J.M., Clayton, D.F., and Greenough, W.T. 1995. Correspondence between sites of NGFI-A induction and sites of morphological plasticity following exposure to environmental complexity. Brain Res. Mol. Brain Res. 32: $211-220$.

Walsh, R.N., Budtz-Olsen, O.E., Penny, J.E., and Cummins, R.A. 1969. The effects of environmental complexity on the histology of the rat hippocampus. J. Comp. Neurol. 137: 361-366.

Wei, F., Li, P., and Zhuo, M. 1999. Loss of synaptic depression in mammalian anterior cingulate cortex after amputation. J. Neurosci. 19: 9346-9354.

Wei, F., Wang, G.D., Kerchner, G.A., Kim, S.J., Xu, H.M., Chen, Z.F., and Zhuo, M. 2001. Genetic enhancement of inflammatory pain by forebrain NR2B overexpression. Nat. Neurosci. 4: 164 169.

Williams, B.M., Luo, Y., Ward, C., Redd, K., Gibson, R., Kuczaj, S.A., and McCoy, J.G. 2001. Environmental enrichment: Effects on spatial 
memory and hippocampal CREB immunoreactivity. Physiol. Behav. 73: 649-658.

Wu, M.F., Pang, Z.P., Zhuo, M., and Xu, Z.C. 2005a. Prolonged membrane potential depolarization in cingulate pyramidal cells after digit amputation in adult rats. Mol. Pain 1: 23.

Wu, L.J., Toyoda, H., Zhao, M.G., Lee, Y.S., Tang, J., Ko, S.W., Jia, Y.H., Shum, F.W., Zerbinatti, C.V., Bu, G., et al. 2005b. Upregulation of forebrain NMDA NR2B receptors contributes to behavioral sensitization after inflammation. J. Neurosci. 25: 11107-11116.

Wu, L.J., Zhao, M.G., Toyoda, H., Ko, S.W., and Zhuo, M. 2005c. Kainate receptor-mediated synaptic transmission in the adult anterior cingulate cortex. J. Neurophysiol. 94: 1805-1813.

Zhao, M.G., Toyoda, H., Lee, Y.S., Wu, L.J., Ko, S.W., Zhang, X.H., Jia,
Y., Shum, F., Xu, H., Li, B.M., et al. 2005. Roles of NMDA NR2B subtype receptor in prefrontal long-term potentiation and contextual fear memory. Neuron 47: 859-872.

Zhao, M.G., Ko, S.W., Wu, L.J., Toyoda, H., Xu, H., Quan, J., Li, J., Jia, Y., Ren, M., Xu, Z.C., et al. 2006. Enhanced presynaptic neurotransmitter release in the anterior cingulate cortex of mice with chronic pain. J. Neurosci. 26: 8923-8930.

Zhuo, M. 2004. Central plasticity in pathological pain. Novartis Found. Symp. 261: 132-145. discussion 145-154.

Received January 11, 2007; accepted in revised form February 8, 2007. 


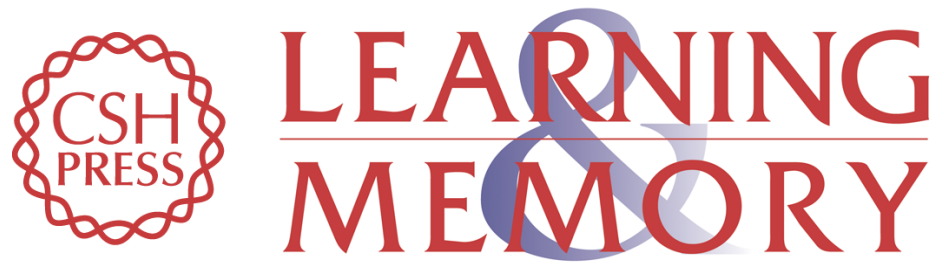

\section{Alteration of cingulate long-term plasticity and behavioral sensitization to inflammation by environmental enrichment}

Fanny W.F. Shum, Long-Jun Wu, Ming-Gao Zhao, et al.

Learn. Mem. 2007, 14:

Access the most recent version at doi:10.1101//m.530607

References This article cites 65 articles, 16 of which can be accessed free at: http://learnmem.cshlp.org/content/14/4/304.full.html\#ref-list-1

License

Email Alerting Receive free email alerts when new articles cite this article - sign up in the box at the Service top right corner of the article or click here. 